\title{
Kramers Problem for a Multiwell Potential
}

\author{
M. Arrayás, ${ }^{1,2}$ I. Kh. Kaufman, ${ }^{1,3}$ D. G. Luchinsky, ${ }^{1,3}$ P. V. E. McClintock, ${ }^{1}$ and S. M. Soskin ${ }^{4}$ \\ ${ }^{1}$ Department of Physics, Lancaster University, Lancaster LA1 4YB, United Kingdom \\ ${ }^{2}$ Instituut-Lorentz, Universiteit Leiden, Postbus 9506, 2300 RA Leiden, The Netherlands \\ ${ }^{3}$ Russian Research Institute for Metrological Service, Ozernaya 46, 119361 Moscow, Russia \\ ${ }^{4}$ Institute of Semiconductor Physics, Ukrainian National Academy of Sciences, Kiev, Ukraine
}

(Received 22 September 1999)

\begin{abstract}
Fluctuational escape from a multiwell potential is shown to display new features, as compared to the conventional single-well case. The flux $J$ may depend on friction $\Gamma$ exponentially strongly, over an exponentially long period; for small enough temperatures, $J(\Gamma)$ undergoes marked oscillations in the range of small $\Gamma$, and the time evolution of $J$ changes drastically as $\Gamma$ exceeds a critical value.
\end{abstract}

PACS numbers: 05.40.-a, 02.50.-r, 05.20.-y

In his celebrated work [1], Kramers considered noiseinduced escape from a single metastable potential well. His principal result was that, after a short period of time during which quasiequilibrium is formed within the well, the escape flux decays exponentially,

$$
J=\alpha e^{-\alpha t},
$$

where the escape rate $\alpha$ is the product of an Arrhenius factor [2] $\exp (-\Delta U / T)$ and a preexponential factor that depends only weakly on temperature $T$, friction $\Gamma$, and the details of the potential $U(q)$,

$$
\alpha=A(T, \Gamma,[U]) \exp \left(-\frac{\Delta U}{T}\right), \quad T \ll \Delta U .
$$

Kramers derived explicit formulas for $A$ in the ultraunderdamped and moderate-to-overdamped limits.

In the ensuing developments and generalizations of the Kramers problem [3,4] (see [5] and [6] for some recent developments), there were two activities that are of present relevance. The first was the problem of filling the "gap" between the ultra-underdamped and moderate-tooverdamped limits of the expressions for the preexponential factor $A$. This activity was crowned by the work by Mel'nikov [7]. The second activity includes recent studies $[8,9]$ of the flux dynamics during a short initial period while quasiequilibrium in the well is forming.

The subject of our paper is the escape flux from a multiwell metastable potential during the period preceding the formation of quasiequilibrium, which takes exponentially longer than in the single-well case. This will in practice be the only relevant time scale for low temperatures where the quasistationary stage (1) is never attained. We emphasize also that our discussion relates to exponentially strong effects rather than just to a (weak) preexponential factor. The results are immediately applicable to interwell transition rates in stable potentials with more than two wells, e.g., to rf SQUIDs [10], ionic channels [11], and numerous other systems in nature that can be described as classical multiwell potential systems subject to a linear friction and white noise.
Figure 1 presents the results of a computer simulation of the escape (first-passage) flux. The system is put initially into the bottom of the well 1 [12] [i.e., $q(0)=q_{1}, \dot{q}(0)=$ $0]$ and then follows the stochastic equation,

$$
\begin{gathered}
\ddot{q}+\Gamma \dot{q}+d U / d q=f(t), \\
\langle f(t)\rangle=0, \quad\left\langle f(t) f\left(t^{\prime}\right)\right\rangle=2 \Gamma T \delta\left(t-t^{\prime}\right), \\
U(q)=\left\{\begin{array}{l}
0.06(q+1.5)^{2}-\cos (q) \text { at } q<q_{l}=4.5 \\
-\infty \text { at } q>q_{l}
\end{array}\right.
\end{gathered}
$$

until either the coordinate limit $q_{l}$ (equivalent to an absorbing boundary) or the time limit $t_{l}=1000$ is reached [13], after which it is reset to the bottom of the well 1 and everything is repeated. Once the statistics are deemed adequate, we calculate the flux

$$
J(t) \equiv \frac{1}{N_{\text {reset }}} \frac{\Delta N(t)}{\Delta t}
$$

as a function of time. Here, $N_{\text {reset }}$ is the overall number of resettings, and $\Delta N(t)$ is the number of escapes during the interval $[t, t+\Delta t]$ (the escape is defined as a reaching $q_{l}$ ), where $\Delta t$ is chosen to be much larger than the typical interval between two successive escapes, but much smaller than the characteristic time scales over which the flux (4) may change significantly.

One can resolve in Fig. 1(b) three distinct stages: a rapid initial growth of $J$ on a time scale $t_{\text {in }}$ (related to the formation of quasiequilibrium within the initial well), followed by a slow decay with a characteristic decay time $t_{s} \gg t_{\text {in }}$ (related to the formation quasiequilibrium between wells 1 and 2), and then a yet slower exponential (quasistationary) decay with a decay time $t_{q s} \gg t_{s}$ [where, on the scale of the figure, $J(t)$ appears constant].

Given the hierarchy $t_{\text {in }} \ll t_{s} \ll t_{q s}$, the processes of interattractor [14] transitions may be considered at the second and third stages as being instantaneous [15] and, correspondingly, one may readily use the approximation of kinetic equations for the well populations $W_{i}$, with constant interattractor transition rates $\alpha_{i j}$. These equations are easily solved and the flux is 

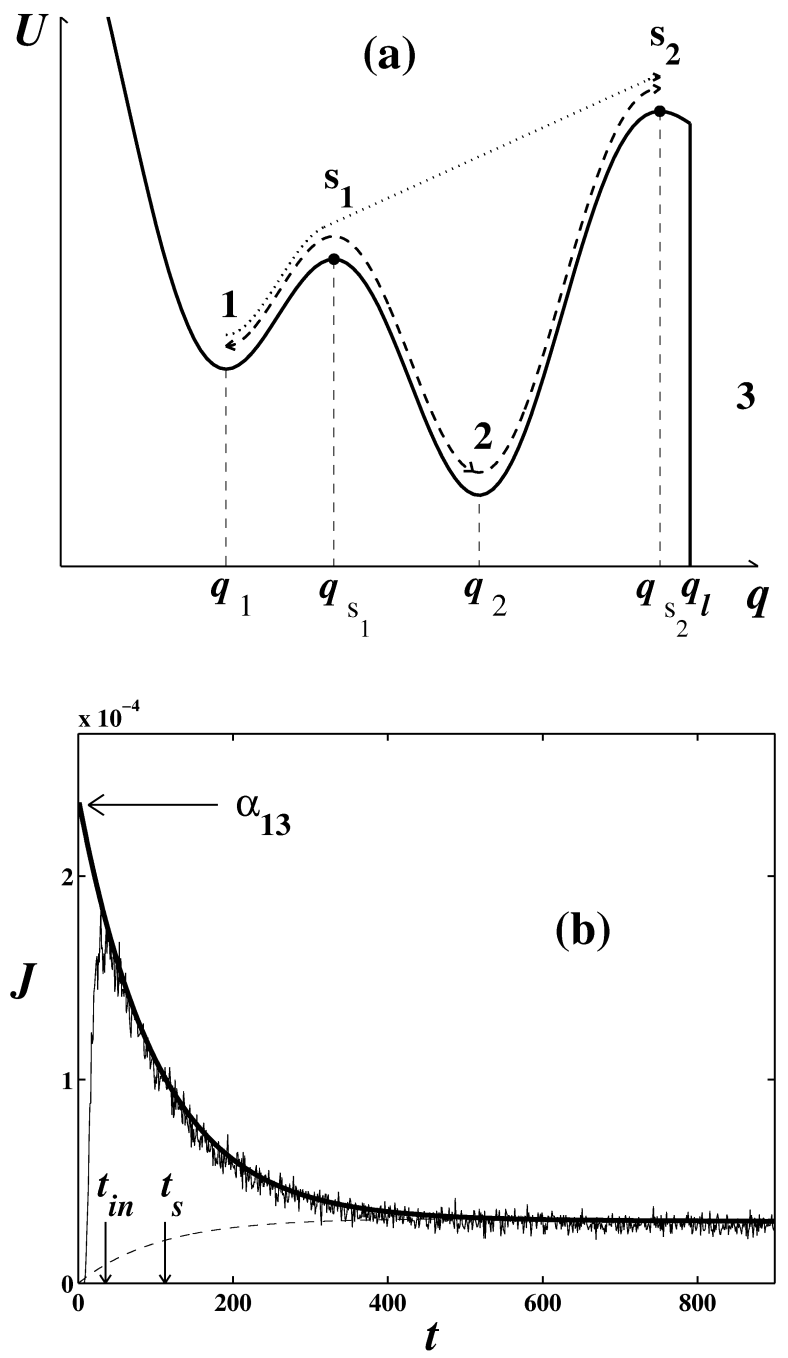

FIG. 1. (a) The potential (3) and a sketch of direct (dotted line) and indirect (dashed line) escape paths $1 \rightarrow \mathrm{s}_{2}$; thin dashed lines indicate positions of the local minima $\left(q_{1}, q_{2}\right)$ and maxima $\left(q_{s 1}, q_{s 2}\right)$; (b) simulations of a dependence of the escape flux on time $J(t)$ (thin line) for the model (3) at $\Gamma=0.15, T=0.4$. The thick solid and dashed lines show the approximation of $J(t)$ by Eq. (5) in which $\alpha_{12}, \alpha_{21}$, and $\alpha_{q s}$ are calculated by the Kramers-Melnikov formula [4], and, for the thick solid line, $\quad \alpha_{13,23}=\alpha_{q s}\left(1+\left\{\Omega_{1} \Omega_{2}^{-1} \exp \left[\left(U_{1}-U_{2}\right) / T\right]\right\}^{ \pm 1}\right) /(1+$ $\left.\left\{m \exp \left[k S_{\min }\left(\mathrm{s}_{2} \rightarrow \mathrm{s}_{1}\right) / T\right]\right\}^{ \pm 1}\right)$, where $\Omega_{1,2}$ are the frequencies of eigenoscillation in the bottom of wells 1,2 , respectively, $k$ is equal to $1,-1$ for the ranges $\Gamma$ providing $\mathrm{s}_{2} \stackrel{\mathrm{nf}}{\rightarrow} 2,1$, respectively, $S_{\min }\left(\mathrm{s}_{2} \rightarrow \mathrm{s}_{1}\right)$ is calculated by the theory [17], and $m$ is the only adjustable parameter ( $m \approx 1.1$ for these parameters); for the dashed line, $\alpha_{13}=0$ and $\alpha_{23}=\alpha_{q s}\left(1+\alpha_{21} / \alpha_{12}\right)$.

$$
\begin{gathered}
J(t)=\alpha_{13} e^{-t / t_{s}}+\alpha_{q s}\left(e^{-t / t_{q s}}-e^{-t / t_{s}}\right), \\
t_{s} \approx \alpha_{12}^{-1}, \quad t_{q s} \approx \alpha_{q s}^{-1} \approx \alpha_{12} /\left(\alpha_{12} \alpha_{23}+\alpha_{21} \alpha_{13}\right), \\
T \ll U_{\mathrm{s}_{1}}-U_{1}, \quad t \gg t_{\mathrm{in}} .
\end{gathered}
$$

Thus, in order to know the flux dynamics, one needs to find $\alpha_{i j}$. Coefficients $\alpha_{12}, \alpha_{21}$ and the quasistationary flux $\alpha_{q s}$ can be calculated from the Kramers-Melnikov formula [4]. Thus, only one of the four $\alpha_{i j}$ coefficients needs to be found independently. We choose it to be $\alpha_{13}$.
The theoretical problem of finding $\alpha_{13}$ is inherently difficult. The Melnikov method is generally not valid in the multiwell case [4]. Therefore, the method of optimal fluctuation (see, e.g., [16]) was suggested [17], seeking the escape rate in the form

$$
\alpha_{13}=P e^{-S_{\min } / T},
$$

where the action $S_{\min }$ does not depend on $T$ and the dependence of the prefactor $P$ on $T$ is relatively weak.

In order to find $S_{\min }$, one must find the minimum of certain functional $[17,18]$

$$
\begin{gathered}
S_{\min } \equiv S_{\min }\left(1 \rightarrow \mathrm{s}_{2}\right)=\min _{[q(t)], t_{t r}}(S), \\
S \equiv S_{t_{t r}}[q(t)]=\frac{1}{4 \Gamma} \int_{0}^{t_{t r}} d t(\ddot{q}+\Gamma \dot{q}+d U / d q)^{2} \\
q(0)=q_{1}, \quad \dot{q}(0)=0, \quad q\left(t_{t r}\right)=q_{\mathrm{s}_{2}}, \quad \dot{q}\left(t_{t r}\right)=0 .
\end{gathered}
$$

The trajectory $[q(t)]$ providing minimal $S$ does not include attractor 2. It is called [17] the most probable direct transition path (MPDTP). The main features of $S_{\min }$ and the MPDTP are illustrated in Figs. 2 and 3 for (3); see [17] for a rigorous general treatment [19].

From Fig. 2, where the dependence of the excess action

$$
\Delta S \equiv \Delta S\left(1 \rightarrow \mathrm{s}_{2}\right)=S_{\min }\left(1 \rightarrow \mathrm{s}_{2}\right)-\left(U_{\mathrm{s}_{2}}-U_{1}\right)
$$

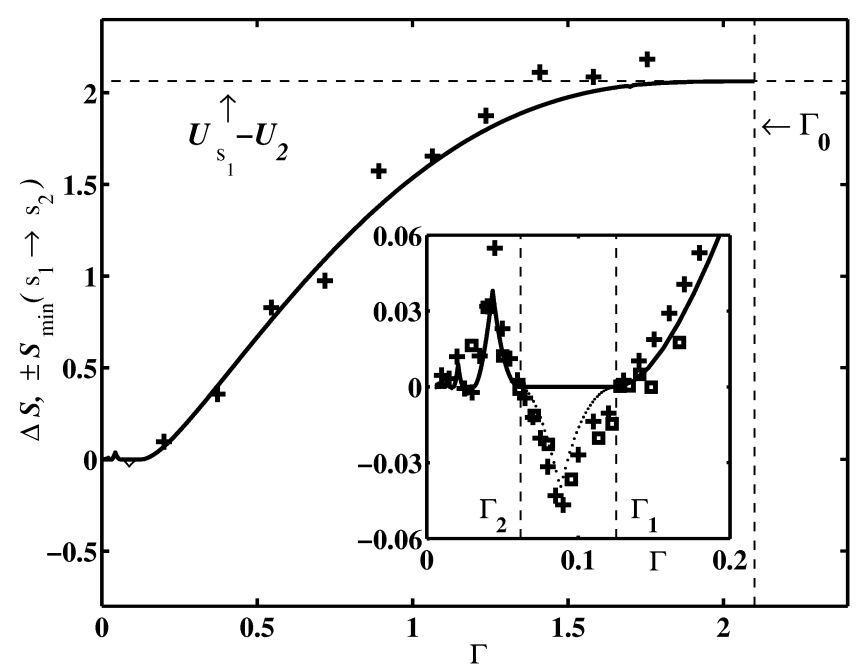

FIG. 2. Theoretical and experimental data on direct escapes/ transitions in the metastable potential (3) [Fig. 1(a)]. The calculated excess of action (related to the escape rate $\alpha_{13}$ ) over a difference of energies, $\Delta S\left(1 \rightarrow \mathrm{s}_{2}\right)$, (8) is shown by the solid line. The calculated $\pm S_{\min }\left(\mathrm{s}_{2} \rightarrow \mathrm{s}_{1}\right)$, related to $R$ (11) by Eq. (12), is shown by the dotted line (it overlaps the solid line in the half-plane of positive ordinates). The corresponding quantity (13) based on data obtained by electronic and computer simulations is shown by squares and crosses, respectively. Values $\Gamma_{n \geq 1}$ correspond to noise-free saddle connections with $n-1$ turning points. At $\Gamma=\Gamma_{0}=2 \Omega_{2} \approx 2.1$, the turning points in the noise-free trajectories $\mathrm{s}_{2} \stackrel{\mathrm{nf}}{\rightarrow} 2$ and $\mathrm{s}_{1} \stackrel{\mathrm{nf}}{\rightarrow} 2$ disappear. The inset shows the low $\Gamma$ range enlarged. 

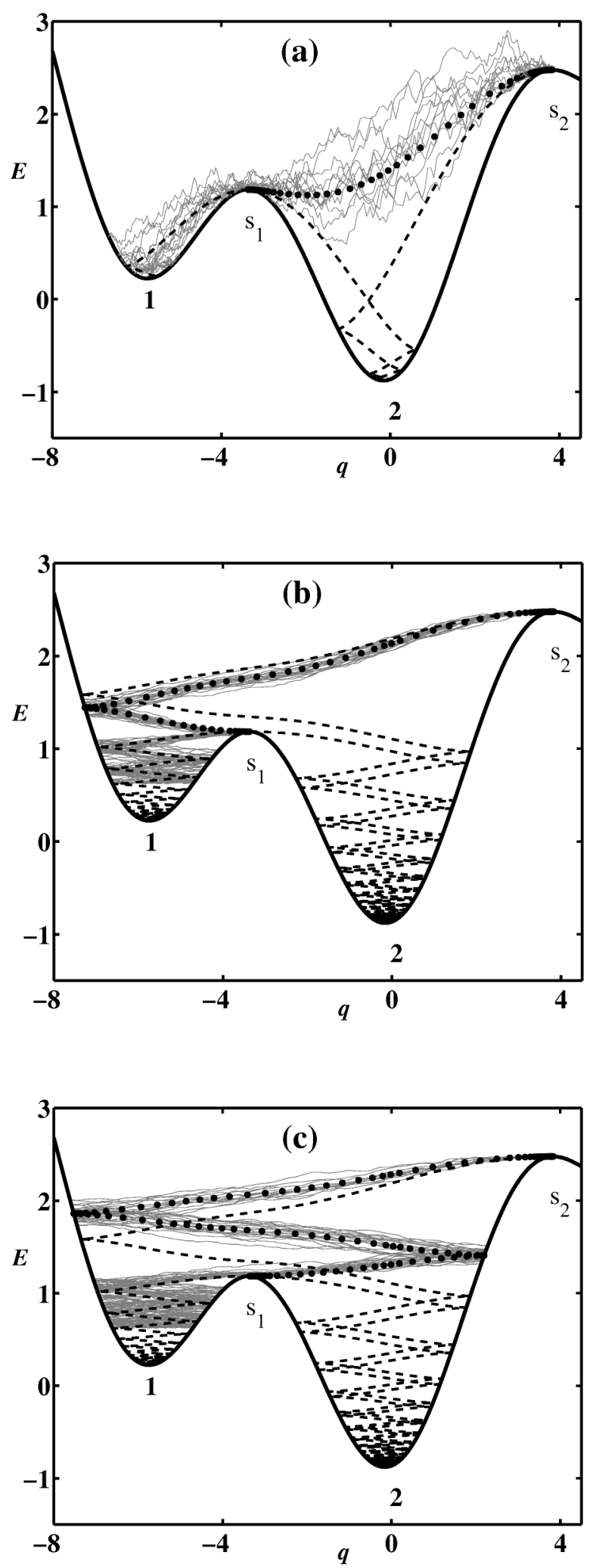

FIG. 3. Simulated direct transition paths $s_{2} \rightarrow 1$ (thin solid lines) in the energy-coordinate plane $E-q$ [where $E=\dot{q}^{2} / 2+U(q)$ ], corresponding to Eq. (3) at different $\Gamma$ : (a) 0.5 , (b) 0.05 , and (c) 0.04 [ $T=0.05$ for (a) and $T=0.005$ for (b) and (c)]. The noise-free trajectories $\mathrm{s}_{2} \stackrel{\mathrm{nf}}{\rightarrow} 2$ and $\mathrm{s}_{1} \stackrel{\mathrm{nf}}{\rightarrow} 1,2$ are shown by dashed lines. The MPDTPs $\mathrm{s}_{2} \rightarrow \mathrm{s}_{1}$ are shown by thick dotted lines. on $\Gamma$ is considered over the whole range of $\Gamma$ from very strong damping to the ultra-underdamped case, one can resolve three distinct regions of $\Gamma$.

In the overdamped region, $\Gamma \geq \Gamma_{0}=2 \Omega_{2}$ (where $\Omega_{2}$ is the frequency of eigenoscillation in the bottom of well 2 ), there is no MPDTP $1 \rightarrow \mathrm{s}_{2}$ at all so that $\alpha_{13}=0$.

In the moderate-friction region $\left[\Gamma_{1}, \Gamma_{0}\right], \Delta S(\Gamma)$ is monotonic and undergoes the largest variation: from 0 to $U_{\mathrm{s}_{1}}-U_{2}$. The MPDTP [see Fig. 3(a)] is the time reversal of the trajectory $\mathrm{s}_{2} \stackrel{A=A_{-}}{\rightarrow} \mathrm{s}_{1} \stackrel{\mathrm{nf}}{\rightarrow} 1$. The latter is just the noise-free relaxation from $s_{1}$ to 1 , whereas the former is the solution of (cf. also [18])

$$
\begin{gathered}
\ddot{q}_{d}+\Gamma \frac{1+A e^{\Gamma t}}{1-A e^{\Gamma t}} \dot{q}_{d}+d U\left(q_{d}\right) / d q_{d}=0, \\
q_{d}(0)=q_{\mathrm{s}_{2}}, \quad \dot{q}_{d}(0)=0,
\end{gathered}
$$

where $A=A_{-}$is a negative constant providing for the minimal $S$ among all values of $A$ for which $\left[q_{d}(t)\right]$ reaches $\mathrm{s}_{1}$ [in general, there may be an infinite set of $A$ providing $\left[q_{d}(t)\right]$ connecting the saddles: the corresponding trajectories differ by their number of turning points].

The underdamped region, $\Gamma \leq \Gamma_{1}$, is divided by characteristic values of friction, $\Gamma_{n \geq 1}$. Each value $\Gamma_{n}$ provides for the noise-free saddle connection $\mathrm{s}_{2} \stackrel{\mathrm{nf}}{\rightarrow} \mathrm{s}_{1}$ and the latter possesses $n-1$ turning points. In this region, $\Delta S(\Gamma)$ undergoes oscillations which correspond to an alternation between two situations. In the first, $\left[\Gamma_{2 m}, \Gamma_{2 m-1}\right](m \geq 1)$, a noise-free trajectory $\mathrm{s}_{2} \stackrel{\mathrm{nf}}{\longrightarrow} 1$ exists and the MPDTP is just its time reversal, with $\Delta S=0$. In the second situation, $\left[\Gamma_{2 m+1}, \Gamma_{2 m}\right](m \geq 1)$, the action varies nonmonotonically with $\Gamma$, and has cusps. This is due to a competition between the two paths which are the time reversals, respectively, of $\mathrm{s}_{2} \stackrel{A_{-}}{\rightarrow} \mathrm{s}_{1} \stackrel{\mathrm{nf}}{\rightarrow} 1$ and $\mathrm{s}_{2} \stackrel{A_{+}}{\rightarrow} \mathrm{s}_{1} \stackrel{\text { nf }}{\rightarrow} 1$, where $\mathrm{s}_{2} \stackrel{A_{+}}{\rightarrow} \mathrm{s}_{1}$ are given by the solutions of (9) with $A_{+} \equiv A_{+}(\Gamma)>0$ and $A_{-} \equiv A_{-}(\Gamma)<0$, respectively [see Figs. 3(b) and $3(\mathrm{c})$, respectively]. As $\Gamma$ varies, $S$ along one path becomes equal to $S$ along another, at a certain $\Gamma$, leading to switching between the paths and to the cusp in $\Delta S(\Gamma)$ (cf. discontinuities in the nonequilibrium potential [18] and fluctuational separatrix [16]).

Thus, [17] predicts an exponentially strong dependence on friction (including such nontrivial features as oscillations and cusps) for the escape rate $\alpha_{13}$ at $t \gg t_{\text {in }}$. To establish whether these (and the properties of MPDTPs described above) occur in reality, we have undertaken analog electronic [20] and computer [21] simulations. A necessary condition is smallness of the temperature: $T \ll$ $\Delta S,\left(U_{\mathrm{s}_{1}}-U_{1}\right)$. However, to obtain reasonable statistics at such a small temperature would require an unrealistically long time $\left(\propto \exp \left[\left(U_{\mathrm{s}_{2}}-U_{1}+\Delta S\right) / T\right]\right)$ [22]. We have overcome this difficulty by exploiting the property of detailed balance [23], which implies [17] that the MPDTP $\mathrm{s}_{2} \rightarrow 1$ is just the time reversal of the MPDTP $1 \rightarrow \mathrm{s}_{2}$, with the corresponding actions differing by $U_{\mathrm{s}_{2}}-U_{1}$, i.e., 


$$
\begin{aligned}
\Delta S\left(1 \rightarrow \mathrm{s}_{2}\right) & =S_{\min }\left(\mathrm{s}_{2} \rightarrow 1\right) \\
& =\left\{\begin{array}{cl}
0 & \text { at } \mathrm{s}_{2} \stackrel{\mathrm{nf}}{\rightarrow} 1 \\
S_{\min }\left(\mathrm{s}_{2} \rightarrow \mathrm{s}_{1}\right) & \text { at } \mathrm{s}_{2} \stackrel{\mathrm{nf}}{\rightarrow} 2
\end{array}\right.
\end{aligned}
$$

so that information about the transition $s_{2} \rightarrow 1$ is equivalent to that for $1 \rightarrow s_{2}$, but the experimental time required is of course much smaller in the former case $[\propto \exp (\Delta S / T)]$ than in the latter.

Figure 3(a) demonstrates that, for $\Gamma \in\left[\Gamma_{1}, \Gamma_{0}\right]$, most of the direct paths $s_{2} \rightarrow 1$ do indeed concentrate near $\mathrm{s}_{2} \stackrel{A_{-}}{\rightarrow} \mathrm{s}_{1} \stackrel{\mathrm{nf}}{\rightarrow} 1$. Figures 3 (b) and 3(c) demonstrate switching of the MPDTP from $\mathrm{s}_{2} \stackrel{A_{+}}{\rightarrow} \mathrm{s}_{1} \stackrel{\text { nf }}{\rightarrow} 1$ to $\mathrm{s}_{2} \stackrel{A_{-}}{\rightarrow} \mathrm{s}_{1} \stackrel{\mathrm{nf}}{\rightarrow} 1$ as $\Gamma$ decreases in the range $\left[\Gamma_{3}, \Gamma_{2}\right]$.

In order to study $S_{\min }$ we use the following technique. The system is put at $s_{2}$ and then follows its stochastic dynamics (3) until either the bottom of one of the wells is approached or the coordinate $q_{l}$ is reached. After that, the system is reset to $s_{2}$ and the operation is repeated. Once adequate statistics have been obtained, we calculate the ratio of transitions to the wells 1 and 2 :

$$
R \equiv R(T)=\frac{N_{\mathrm{s}_{2} \rightarrow 1}}{N_{\mathrm{s}_{2} \rightarrow 2}} .
$$

It is easy to see that $R \propto \exp \left[ \pm S_{\min }\left(\mathrm{s}_{2} \rightarrow \mathrm{s}_{1}\right) / T\right]$ [where $(+,-)$ correspond to ranges of $\Gamma$ providing $\mathrm{s}_{2} \stackrel{\mathrm{nf}}{\rightarrow} 1,2$, respectively]. So, $S_{\min }\left(\mathrm{s}_{2} \rightarrow \mathrm{s}_{1}\right)$ is related to $R(11)$ as

$$
\pm S_{\min }\left(\mathrm{s}_{2} \rightarrow \mathrm{s}_{1}\right)=\lim _{T \rightarrow 0}\{T \ln [R(T)]\}
$$

where $(+,-)$ correspond to $\mathrm{s}_{2} \stackrel{\mathrm{nf}}{\rightarrow} 1,2$, respectively.

However, there is always a lower limit for $T$ in simulations, $T_{l}$, because the overall simulation time must not become unrealistically long. That is why the use of (12) may, in practice, introduce significant inaccuracy. To reduce the influence of the preexponential factor we measure $R$ both at $T_{l}$ and at a slightly higher temperature, $T_{l}+\Delta T$ $\left[T_{l} \gg \Delta T \Im T_{l}^{2} / S_{\min }\left(\mathrm{s}_{2} \rightarrow \mathrm{s}_{1}\right)\right]$, so that

$$
\pm S_{\min }\left(\mathrm{s}_{2} \rightarrow \mathrm{s}_{1}\right) \approx \frac{T_{l}^{2}}{\Delta T} \ln \left(\frac{R\left(T_{l}+\Delta T\right)}{R\left(T_{l}\right)}\right) \text {. }
$$

The quantities on the left and right of Eq. (13) are shown in Fig. 2, respectively, by the dotted line (theory) and by squares and crosses (electronic and computer simulations respectively). The agreement is satisfactory (given that $5 \gtrless S_{\min } / T_{l} \approx 7$ ).

Note that the magnitude of the largest oscillation in action may significantly exceed $U_{\mathrm{s}_{2}}-U_{1}$. This occurs if the initial well 1 is adjacent to an external saddle $s_{2}$ while its depth is much less than that of the other well.

Finally, we comment on the experimental consequence of the cutoff of the MPDTP, namely, the drastic change of the time evolution of $J$ for $t_{\text {in }} \approx t \ll t_{s}$ : at $\Gamma<\Gamma_{0}$, one may, in principle, make $T$ small enough that the sharp growth of $J(t)$ at $t \gtrless t_{\text {in }}$ turns into a nearly constant value at $t_{\text {in }} \ll t \ll t_{\text {in }} \alpha_{13} /\left(\alpha_{12} \alpha_{23}\right)$, while, at $\Gamma>\Gamma_{0}, J(t) \approx$ $\alpha_{12} \alpha_{23} t$ over the whole relevant time scale [cf. thin solid and dashed lines in Fig. 1(b)]. Another drastic change occurs with the time evolution of the transition flux $s_{2} \rightarrow 1$ : at $\Gamma<\Gamma_{0}$, it has a high narrow peak at $t \approx t_{\text {in }}$, whereas, at $\Gamma>\Gamma_{0}$, it is a monotonically (and very slowly) growing function of $t$, at $t \ll t_{s}$.

This work was supported by the Engineering and Physical Sciences Research Council (UK), and by INTAS.

[1] H. A. Kramers, Physica 7, 284 (1940).

[2] S. Arrhenius, Z. Phys. Chem. (Leipzig) 4, 226 (1889).

[3] P. Hanggi, P. Talkner, and M. Borkovec, Rev. Mod. Phys. 62, 251 (1990).

[4] V. I. Mel'nikov, Phys. Rep. 209, 1 (1991).

[5] D. J. Bicout, A. M. Berezhkovskii, A. Szabo, and G. H. Weiss, Phys. Rev. Lett. 83, 1279 (1999).

[6] R. Reigada, A.H. Romero, K. Lindenberg, and J. M. Sancho, J. Chem. Phys. 111, 676 (1999).

[7] V. I. Mel'nikov, Sov. Phys. JETP 60, 380 (1984).

[8] V. A. Shneidman, Phys. Rev. E 56, 5257 (1997).

[9] S. M. Soskin et al., in Stochaos: Stochastic and Chaotic Dynamics in the Lakes, edited by D. Broomhead, E. Luchinskaya, P. V.E. McClintock, and T. Mullin (AIP, Melville, NY, 2000), p. 60.

[10] R. Barone and G. Paterno, Physics and Applications of the Josephson Effect (Wiley, New York, 1982).

[11] B. Hille, Ionic Channels of Excitable Membranes (Sinauer Associates, Inc., Sunderland, 1992).

[12] The bottom of the well is the most natural initial state because it is a stable state of the noise-free system. But it is not in fact crucial for the flux on the relevant time scale $t \gg t_{\text {in }}$ [see (5)] that the initial state should be at the bottom: the only restriction is that the initial state should lie within a particular well (e.g., well 1).

[13] The choice of $q_{l}, t_{l}$ is quite arbitrary: $q_{l}$ may be chosen between $q_{\mathrm{s}_{2}} \approx 3.8$ and $q_{3} \approx 5.3$, where $q_{3}$ is the coordinate of the local minimum in $\tilde{U}(q)=0.06(q+1.5)^{2}-\cos (q)$ to the right from $\mathrm{s}_{2} ; t_{l}$ is to greatly exceed $t_{s}$ [see (5)] which is equal to $\approx 112$ for the given set of parameters.

[14] For brevity, we refer to region 3 also as an "attractor."

[15] M. I. Freidlin and A. D. Wentzell, Random Perturbations in Dynamical Systems (Springer-Verlag, New York, 1984).

[16] M. I. Dykman, M. M. Millonas, and V.N. Smelyanskiy, Phys. Lett. A 195, 53 (1994).

[17] S. M. Soskin, J. Stat. Phys. 97, 609 (1999).

[18] R. Graham and T. Tel, Phys. Rev. A 33, 1322 (1986).

[19] Some of the results [17] are closely related to some results of [18] which were obtained by a different method and in a different context.

[20] D. G. Luchinsky, P. V.E. McClintock, and M. I. Dykman, Rep. Prog. Phys. 61, 889 (1998).

[21] R. Mannella, in Supercomputation in Nonlinear and Disordered Systems, edited by L. Vázquez, F. Tirando, and I. Martin (World Scientific, Singapore, 1997), pp. 100-130.

[22] Note, however, that preexponential time scales in other systems (e.g., in SQUIDs) may be much smaller, so that the relevant flux may be readily simulated and/or measured.

[23] H. Risken, The Fokker-Planck Equation (Springer-Verlag, Berlin, 1992), 2nd ed. 